\title{
The Effect on Muscle Hardness of Acupuncture Stimulation Using Low-reactive Level Laser Therapy and Silver Spike Point Therapy, along with Stretching
}

\author{
Tadashi Sawada ${ }^{1}$, Nao Tateyama ${ }^{1}$, Takara Ikeda ${ }^{1}$, Keisou Ishimaru ${ }^{2}$ \\ 1: Takarazuka University of Medical and Health Care, Takarazuka, Japan \\ 2: Ryotokuji University Department of Faculty of Health Sciences, Urayasu, Japan
}

\begin{abstract}
Background and aims: Muscle hardness causes lower activity in athletic practice or sport competition. Increase in muscle hardness often cause injury and muscle fatigue. The aim of this study is to investigate the effect on muscle hardness of acupuncture stimulation using low-reactive level laser therapy (LLLT) and silver spike point (SSP) therapy, along with stimulation by stretching.

Materials (Subjects) and Methods: Muscle fatigue was created experimentally, and the effect of LLLT was examined with the indices of muscle hardness and the pennation angle, using ultrasound diagnostic equipment with real-time tissue elastography (RTE) functionality.

Results: As a result, a combined use of stretching and SSP therapy was effective on muscle hardness, while LLLT alone had no immediate lowering of muscle hardness. In addition, only the laser stretching group demonstrated a significant decrease in the pennation angle.

Conclusions: This is because an improved local blood flow due to SSP therapy is considered to have relaxed muscle tonus, which boosted metabolism and removed algogenic substances. This became more effective through a combined use of stretching and low-power laser irradiation. Moreover, it was suggested that stimulation of the acupuncture points in the crus could have a further effect on muscle hardness and the pennation angle.
\end{abstract}

Key words: low-reactive level laser therapy $\bullet$ silver spike point therapy $\bullet$ stretching $\cdot$ muscle hardness • laser acupuncture

\section{Introduction}

Muscle hardness causes lower activity in athletic practice or sport competition. Increase in muscle hardness often causes injury and muscle fatigue, especially after eccentric or heavy loaded exercise. Stretching is one of the effective tools to improve muscle flexibility and physical performance ${ }^{1)}$. However, obtaining accurate information about individual muscle condition is technically difficult for athletes themselves and their trainers and the improvement of muscle hardness is affected by experience. A tissue hardness meter is another way to measure muscle hardness ${ }^{2)}$, however, the influence of skin and fat could not be eliminated. Thus, ultrasound elastography, an imaging method for measuring tissue elasticity using

\section{Addressee for Correspondence}

Tadashi Sawada

Takarazuka University of Medical and Health Care,

1 Hanayashiki- Midorigaoka, Takarazuka-shi, Hyogo 666-0162, Japan

Phone: +81-72-736-8600 E-mail: sawada@tumh.ac.jp an ultrasound diagnostic scanner with special software, is now widely used to measure muscle hardness ${ }^{3)}$.

Real-time Tissue Elastography (RTE) permits real time measurement of the tissue strain based on the principle that softer tissue has more deformation induced by the same external compression and shows larger strain compared to the harder tissue ${ }^{4-6)}$. Strain differences between each element are color-coded according to relative tissue hardness and the elastogram overlaid on the tissue B mode image in RTE. Moreover, quantitative hardness can be measured by comparing the hardness between the tissue of interest and the standard substance.

Pennation angle is defined as the angle between the orientation of muscle fiber fascicles and the attached tendon axis. Increase in pennation angle indicates increase in longitudinal muscle extension and transverse muscle stiffness after eccentric or heavy loaded exercise ${ }^{7,8}$. Increase in pennation angle means muscle stiffness, inflam-

Received date: September 28th, 2019

Accepted date: December 16th, 2019

J-STAGE Advance Publication Date: April 18th, 2020 
matory response and swelling ${ }^{8}$. Thus, we adopted pennation angle as the index of muscle hardness.

Recently, low-reactive level laser therapy (LLLT) has been recognized as a valid medical tool for the treatment of pain, soft tissue damage and muscle fatigue ${ }^{8-12)}$. Silver Spike Point (SSP) therapy, acupuncture-like transcutaneous electrical nerve stimulation, is expected to the valid medical tool for the treatment of pain and muscular tension ${ }^{13,14)}$. We aimed to investigate the effect on experimentally created muscle hardness of acupuncture stimulation using low-reactive level laser therapy (LLLT) and silver spike point (SSP) therapy, along with stimulation by stretching.

\section{Subjects and Methods}

\section{Subjects}

The participants in this study were thirty-one healthy men (mean \pm standard deviation $(S D)$ : age, $20.3 \pm 1.2$

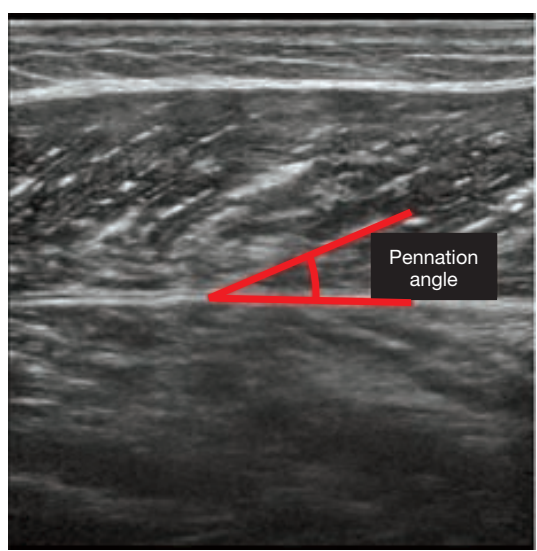

Figure 1: Measurement of pennation angle. Pennation angle is defined as the angl between the orientation of a fascicle and the attached tendon axis

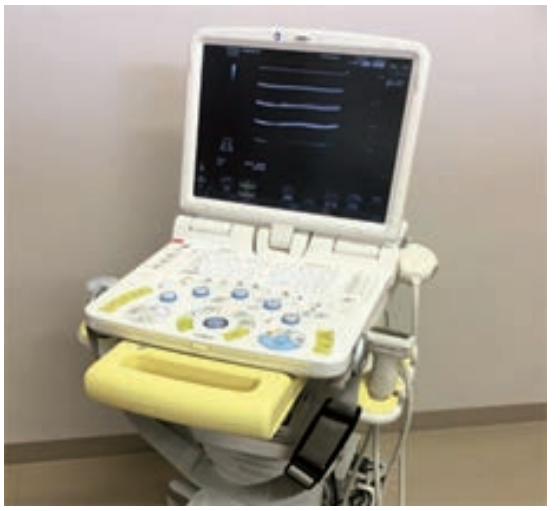

Figure 2: Ultrasound diagnostic scanner. Noblus (Hitachi Aroka Medical, LTD, Tokyo, Japan) and L64 probe $(5-18 \mathrm{MHz})$ were used in this study. years; height, $170.3 \pm 4.8 \mathrm{~cm}$; weight, $63 \pm 6.7 \mathrm{~kg}$ ). The left leg was dominant in 21 participants and the right leg in 10. All participants had not suffered muscle strain in the lower leg and had not performed lower leg resistance training. This study was approved by Ethics Committee of Takarazuka University of Medical Arts and Sciences (No. 1701071) and written consent was obtained from all patients.

\section{Pennation angle and muscle hardness}

Pennation angle and muscle hardness of gastrocnemius muscle were measured.

Pennation angle was measured by the angle between the orientation of a fascicle and the attached tendon axis (Figure 1) with using ultrasound diagnostic scanner (Noblus, Hitachi Aroka Medical, LTD, Tokyo, Japan, Figure 2).

Muscle hardness was measured with using ultrasound diagnostic scanner and L64 probe (5-18 MHz). The measurement of muscle hardness is based on the principle that softer tissue and shows larger strain induced by the same external compression than harder tissues. Strain ratio (muscle/reference ratio; SR) was calculated automatically using built-in software. SR in response to the compression force is smaller in harder tissue than in softer tissue. Thus, we used SR as muscle hardness in this study.

The RTE image was obtained by manual application of repetitive compression with an acoustic coupler (reference material) loaded probe. Figure 3 shows RTE image, B-mode image superimposed by the analyzed color-coded strain image indicating relative hardness of the region of interest. RTE image indicates strain difference between two regions as color or value, and SR is calculated automatically (Figure 4).

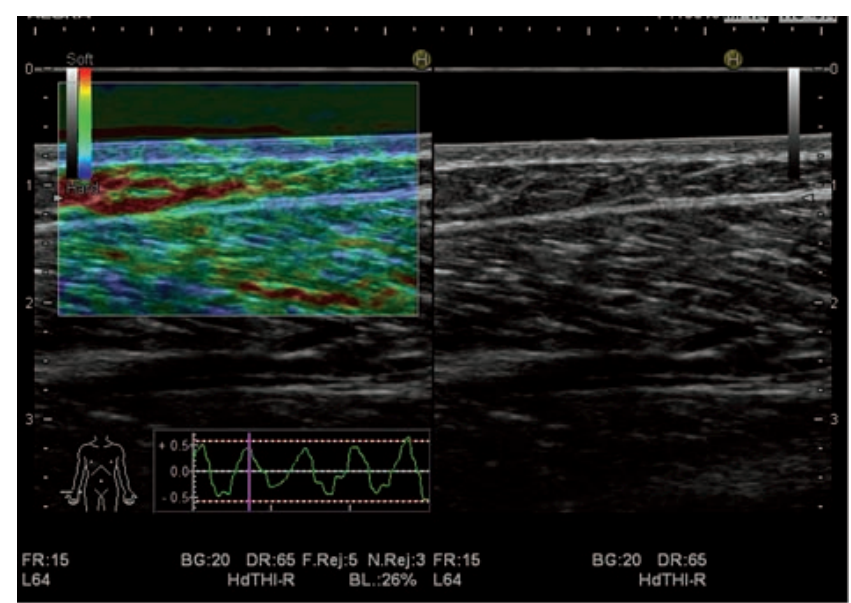

Figure 3: RTE image of Real-time tissue elastography. B-mode image superimposed by the analyzed color-coded strain image indicating relative hardness of the region of interest. 


\section{Experimental design and procedures}

After resting for ten minutes in the experiment room, the participants lay prone on bed and pre-exercise (pre) pennation angle and muscle hardness were measured with ultrasound diagnostic scanner. To make muscle fatigue, the participants performed single-leg ankle plantar-flexion with their dominant leg on an upright position, consisting maximum ankle dorsiflexion for one second and then plantar flexion to the resting position for two seconds. The participants performed 4 sets of 20 repetitions with a two-minute rest between sets. Just after the exercise, post-exercise scans (post) were conducted and then five randomly ordered protocols were assigned. After 25 minutes from the exercise, post25 scans were conducted.

The participants performed five randomly ordered protocols (LA, St, SSP, St-SSP, St-LA) on five separate days
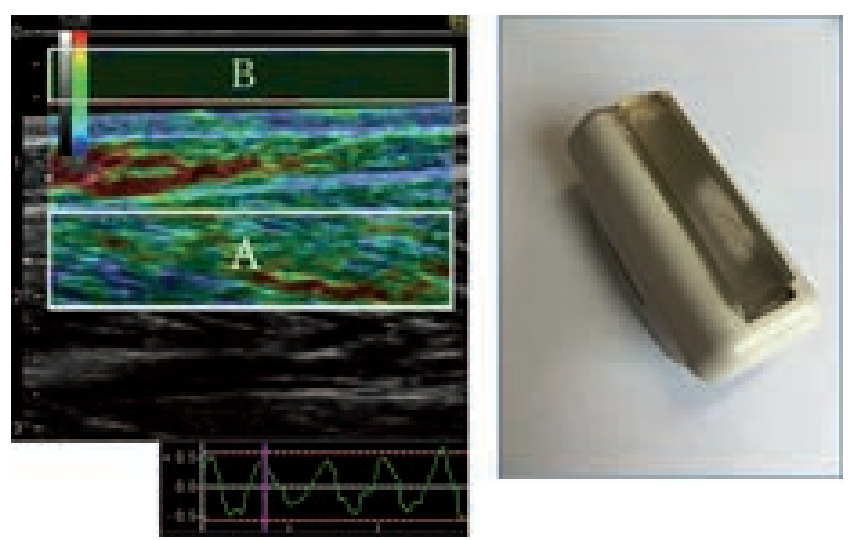

Figure 4: Measurement of Strain Ratio.

a, RTE-image; $\mathbf{b}$, an acoustic coupler attached probe.



Figure 5: Low level laser used in this study. JQ-W1 (Minato Medical Science Co, LTD, Osaka, Japan). at least one week between tests. In LA, the laser used in this protocol was a gallium aluminum, arsenide (Ga-AlAs) diode laser (JQ-W1, Minato Medical Science Co, LTD, Osaka, Japan, Figure 5) giving output power in continuous wave of $180 \mathrm{~mW}$ at a wavelength of $810 \mathrm{~nm}$. The power density of output is $51.4 \mathrm{~W} / \mathrm{cm}^{2}$. The laser was applied to two acupuncture points, Cnengshan (BL-57, International Standard Acupuncture Point indicating upper musculotendon junction of gastrocnemius muscle, Figure 6) and Cnengjin (BL-56, International Standard Acupuncture Point indicating point between medial and lateral gastrocnemius muscle bellies, Figure 6). Both two acupuncture points are clinically applied to Gastrocnemius pain. In St, the participants kept an upright position for three minutes with maintaining their ankles 30-degree dorsiflexion on the stretching board (Figure 7). In SSP, Cnengshan and Cnengjin were stimulated by SSP elec-

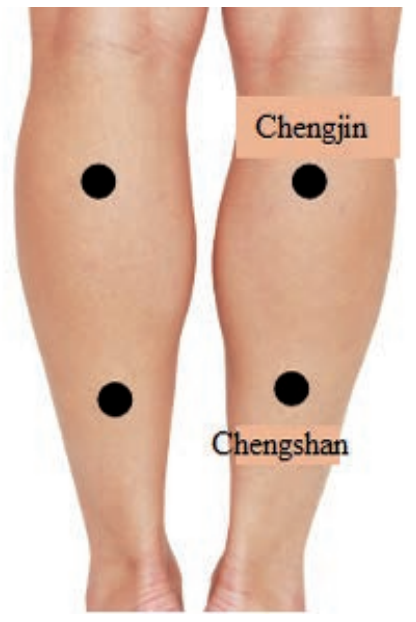

Figure 6: Cnengjin and Cnengshan acupoints.

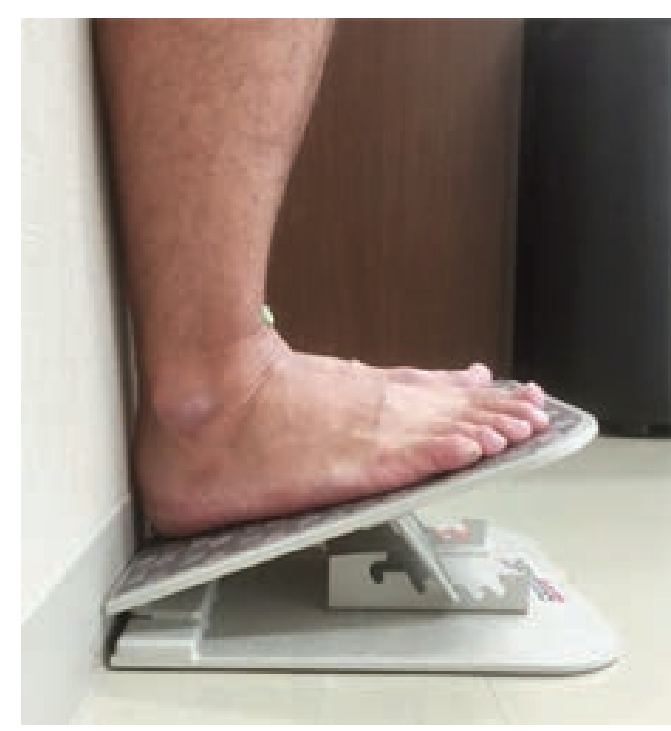

Figure 7: Ankles 30-degree dorsiflexion on the stretching board. 
trodes (Delta 0, Nihon Medix, Kashiwa, Japan, Figure 8) at a wavelength of $110 \mathrm{~Hz}$ for 20 minutes. In St-SSP, St was followed by SSP and in ST-LA, St was followed by LA. All the protocols are illustrated in Figure 9.

\section{Statistics}

After using Friedman test, post-hoc comparisons were performed with Bonferroni-Wilcoxon test. Differences were considered significant when $\mathrm{P}$ was $<0.05$.

\section{Results}

\section{Strain Ratio change in single protocol}

Figure 10 shows the changes in SR performed by three single protocols (LA, St, SSP). The strain ratios in LA were $0.48 \pm 0.12,1.86 \pm 0.25,1.05 \pm 0.13$, in pre, post, post 25 , respectively. The strain ratios in St were $0.64 \pm 0.22,1.92$ $\pm 0.45,0.94 \pm 0.28$, in pre, post, post 25 , respectively. The strain ratios in SSP were $0.53 \pm 0.20,1.85 \pm 0.54,0.44 \pm$ 0.26 , in pre, post, post 25 , respectively. Post strain ratios in

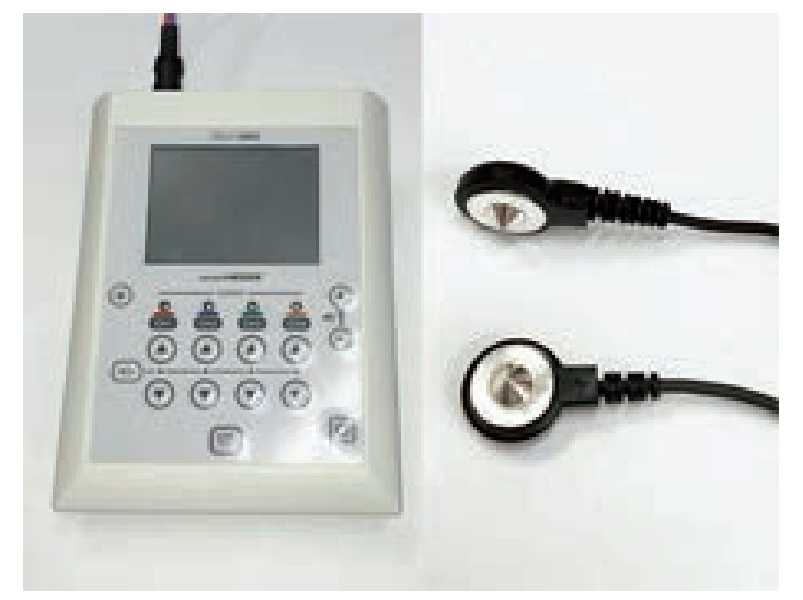

Figure 8: SSP stimulator and electrodes.

Cnengshan and Cnengjin were stimulated by SSP electrodes (Delta 0, Nihon Medix, Kashiwa, Japan) at a wavelength of $110 \mathrm{~Hz}$ for 20 minutes.

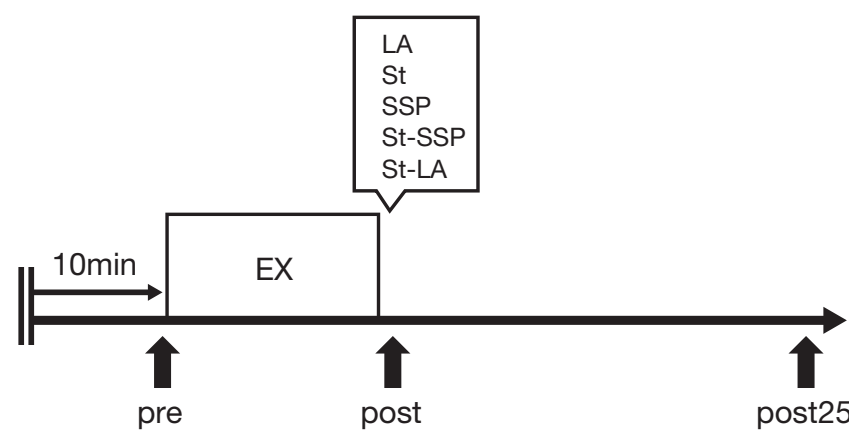

Figure 9: Experimental design and procedures.
LA, St, SSP were significantly higher than the pre strain ratios $(\mathrm{P}<0.05)$. Only the post 25 SR in SSP was significantly lower than the post SR $(\mathrm{P}<0.05)$.

\section{Strain Ratio change in combined protocols}

Figure 11 shows the changes in SR performed by three combined protocols (St-LA, St-SSP). The strain ratios in StLA were $0.49 \pm 0.19,1.52 \pm 0.33,1.10 \pm 0.36$, in pre, post, post 25 , respectively. The strain ratios in St-SSP were 0.47 $\pm 0.22,1.32 \pm 0.40,0.41 \pm 0.16$, in pre, post, post 25 , respectively. Post strain ratios in St-LA, St-SSP were significantly higher than the pre strain ratios $(\mathrm{P}<0.05)$. Only the post25 SR in St-SSP was significantly lower than the

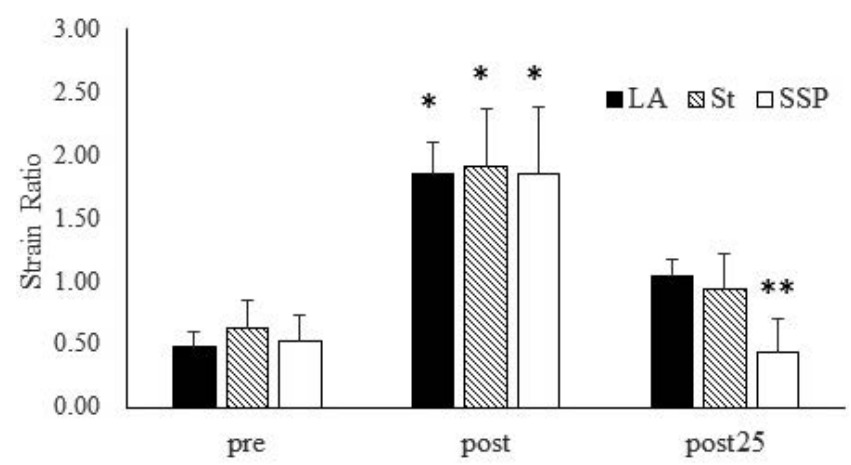

Figure 10: SR change in LA, St and LLA.

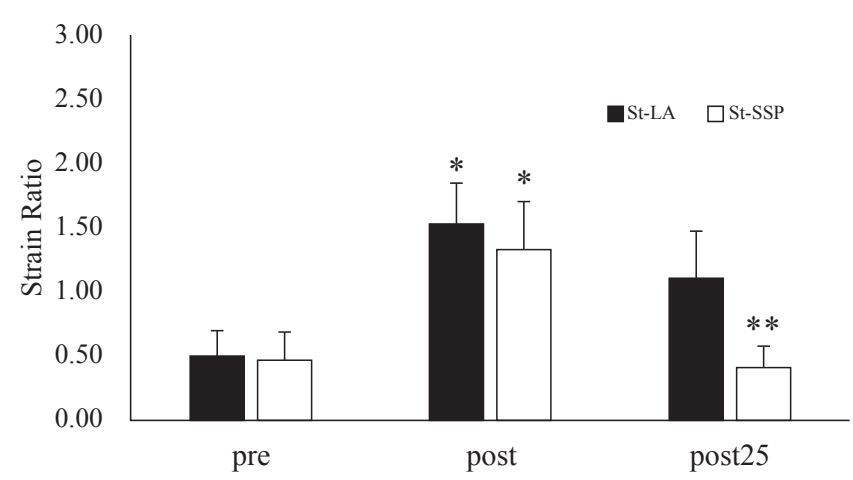

Figure 11: SR change in St-LA, St-SSP.

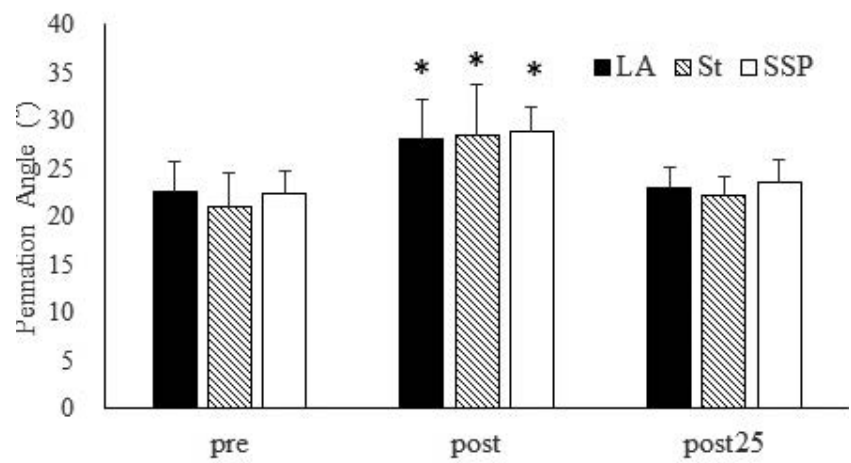

Figure 12: Pennation angle change in LA, St and LLA. 
post SR $(\mathrm{P}<0.05)$.

\section{Pennation angle change in single protocol}

Figure 12 shows the changes in pennation angle performed by three single protocols (LA, St, SSP). The pennation angle in LA were $22.6 \pm 3.1^{\circ}, 28.0 \pm 4.2^{\circ}, 23.0 \pm$ $2.2^{\circ}$, in pre, post, post 25 , respectively. The pennation angle in St were $21.0 \pm 3.5^{\circ}, 28.4 \pm 5.4^{\circ}, 22.2 \pm 1.9^{\circ}$, in pre, post, post 25 , respectively. The pennation angle in SSP were $22.4 \pm 2.4^{\circ}, 28.8 \pm 2.6^{\circ}, 23.6 \pm 2.4^{\circ}$, in pre, post, post 25 , respectively. Post strain ratios in LA, St, SSP were significantly higher than the pre strain ratios $(\mathrm{P}<0.05)$. No post 25 pennation angles were significantly lower than the post SR $(\mathrm{P}<0.05)$.

\section{Pennation change in combined protocols}

Figure 13 shows the changes in pennation angle performed by two combined protocols (St-LA, St-SSP). The pennation angle in St-LA were $24.0 \pm 2.5^{\circ}, 32.8 \pm 4.4^{\circ}$, $24.4 \pm 3.1^{\circ}$, in pre, post, post 25 , respectively. The strain ratios in St-SSP were $23.0 \pm 2.6^{\circ}, 29.2 \pm 4.2^{\circ}, 23.9 \pm 3.0^{\circ}$ in pre, post, post25, respectively. Post strain ratios in St-LA, St-SSP were significantly higher than the pre strain ratios $(\mathrm{P}<0.05)$. Only the post $25 \mathrm{SR}$ in St-LA was significantly lower than the post SR $(\mathrm{P}<0.05)$.

\section{Discussion}

In this study, strain ratio was used to indicate muscle hardness. Post-exercise strain ratios in LA, ST and SSP significantly increased compared to pre-exercise SR's ( $\mathrm{P}<$ $0.05)$. Only the strain ratio in SSP significantly decreased after 25 minutes from the exercise $(\mathrm{P}<0.05)$. Thus, SSP is the only effective to the muscle damage created by repeated eccentric muscle contractions in this study. Acupuncture-like stimulation such as SSP increases regional blood circulation, decreases neuromuscular excitability and promotes relaxation ${ }^{15}$. Thus, SSP revealed more effective to the muscle damage in this study. Post-exercise strain ratios in St-LA and St-SSP also increased significant-

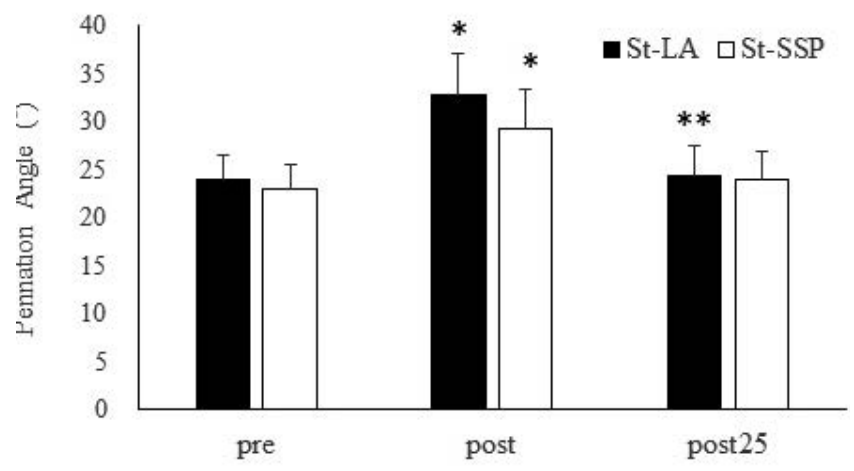

Figure 13: Pennation angle change in St-LA, St-SSP. ly $(\mathrm{P}<0.05)$ and slightly lower than those in LA, ST and SSP. Only the strain ratio in St-SSP significantly decreased after 25 minutes from the exercise $(P<0.05)$. These results that single application of LLLT showed no effect on the strain ratio in this study. Our exercise protocols were so eccentric that single application of LLLT showed less effect than we expected.

For measurements of pennation angle, the orientation of the transducer is important to achieve accurate measurements. Some degrees of misalignments are usually made. Bolsterlee et al reported that accurate pennation angle measurements are obtained when the transducer oriented perpendicular to the skin ${ }^{16)}$. Thus, we used this protocol. Post-exercise pennation angles in LA, ST and SSP significantly increased compared to pre-exercise ones $(\mathrm{P}<0.05)$. No pennation angles in these three conditions significantly decreased after 25 minutes from the exercise. Post-exercise strain ratios in St-LA and St-SSP also increased significantly $(\mathrm{P}<0.05)$. Only the pennation angle in St-LA significantly decreased after 25 minutes from the exercise $(\mathrm{P}<0.05)$.

Low-reactive level laser therapy is effectively applied to reduction of pain, inflammation and muscle tone. LLLT may cause local vascular dilation through stimulating the parasympathetic nervous system ${ }^{17)}$. The mechanisms underlying the effect of LLLT are incompletely understood. However, LLLT is clinically applied to acute pain with radiating mainly on tender points and to chronic or neuropathic pain with radiating stellate ganglion or acupuncture points ${ }^{18-21)}$. As is mentioned before, SSP is cupuncture-like stimulation which increases regional blood circulation, decreases neuromuscular excitability and promotes relaxation ${ }^{15}$. These effects on acupuncture points with SSP electrodes were greater than those with LLLT because SSP electrodes directly touched on acupuncture points and might stimulate autonomic neural pathways 15). The effects of LLLT on acupuncture points are unknown, however, another project to clarify these effects is under progress.

\section{Conclusions}

This study examined the effects of acupuncture stimulation on muscle hardness with using LLLT, SSP therapy, by stretching. SSP and St-SSP significantly reduced muscle hardness $(\mathrm{P}<0.05)$ and ST-SSP was most effective on reducing muscle hardness. LLLT alone had no acute effect on reducing muscle hardness. Only ST-LA reduced pennation angel $(\mathrm{P}<0.05)$. St-LA was most effective on increase in longitudinal muscle extension. 


\section{References}

1: Nakamura K, Kodama T, Mukaino Y (2014): Effects of active individual muscle stretching on muscle junction. J Phys Ther Sc, 26: 341-344.

2: Hiroshi Watanabe Jun Matsuoka J, Nashimoto S, Koga Y, Omori G, Endo K, Tanaka M, Nawata A, Sasaki R (2013): Effective duration of static stretching by the examination of the quadriceps tension degree change with the lower limbs muscular strength measurement. Kouseirenishi, 22: $34-38$.

3: Ueyama H, Taniguchi G, Watanabe S, Oki S, Kiriyama Y, Imai H Toyama Y, Matsumoto H, Nagura T (2014): Ultrasonographic measurement of the vastus medialis muscle geometry, deformity and elasticity before and after static stretching. Japanese Journal of Clinical Biomechanics 35 : 325-329.

4: Akagi, R, Takahashi, H (2014): Effect of a 5-week static stretching program on hardness of the gastrocnemius muscle. Scandinavian Journal of Medicine \& Science in Sports 24: $950-957$.

5: Niitsu M, Michizaki A, Endo A, Takei H, Yanagisawa O (2011): Muscle hardness measurement by using ultrasound elastography: a feasibility study. Acta Radiol, 52: 99-105.

6: Yanagisawa O, Niitsu M, Kurihara T, Fukubayashi T (2011): Evaluation of muscle hardness after exercise with an ultrasound Real-time Tissue Elastography. Japanese Journal of Clinical Sports Medicine 19: 127-131.

7: Inami T, Kawakami Y. (2016): Assessment of individual muscle hardness and stiffness using ultrasound elastography. J Phy Fitness Sports Med, 5: 313 - 317.

8: Margaritelis NV, Theodorou AA, Baltzopoulos V, Maganaris CN, Paschalis V, Kyparos A, Nikolaidis MG. (2015): Muscle damage and inflammation after eccentric exercise: can the repeated bout effect be removed? Physiol Rep, 3: e12648.

9: Hosokawa T, Kawabata Y (2012): Pain treatment of low reactive level laser (LLLT). Masui, 62: 718-727.

10: Sawada T, Ishimaru K (2010): Combined Effects of laser therapy and acupuncture on sports injuries. Journal of Japan Laser therapy Association, 9: 59-62.

11: Sawada T (2016): The effect of distal area stimulation of delayed onset muscle soreness - A comparison of effects between acupuncture stimulation and low reactive level light treatment. Journal of Japan Laser therapy Association, 14:
68-71.

12: Ishimaru K, Sawada T (2014): The documents investigation into low reactive level laser therapy for the delayed onset muscle soreness and trial of the technique of the oriental medicine. Journal of Japan Laser therapy Association, 13: 53-56.

13: Sugimoto K, Konda T, Shimahara M, Hyodo M, Kitade T (1995): A clinical study on SSP (silver spike point) electro-therapy combined with splint therapy for temporo-mandibular joint dysfunction. Masui, Acupunct Electrother Res, 20: 7-13.

14: Ishimaru K (2013): Effects of transcutaneous electrical nerve stimulation (silver spike point : SSP therapy) on the deep pain threshold and the presence of endogenous analgesic substance. Manseitotsu, 32: 39-44.

15: Weerapong P, Hume PA, Kolt GS. (2005): The mechanisms of massage and effects on performance, muscle recovery and injury prevention. Sports Medicine, 35: 235 - 256.

16: Bolsterlee K, Gandevia SC, Herbert RD. (2016): Effect of transducer orientation on errors in ultrasound image-based measurements of human medicalgastrocnemius muscle fascicle length and pennation. Plos ONE, DOI: 10.1371.

17: Kamikawa K, Ohnishi T, Suzuki M, Kanetani G, Shibamoto H. (1982): Laser therapy of pain. J Japan Soc Laser Med, 3: $345-348$.

18: Sawada T, Ishimaru K (2013): Preventive effects of low reactive light treatment applied to different sites against delayed-onset muscle soreness. Journal of Japan Laser therapy Association, 12: 35-38.

19: Morimoto Y (2018): Low reactive level laser therapy (LLLT) for sports injury. Journal of Japan Laser therapy Association, 38: 446-449.

20: Kondo Y, Hirose N, Maeda T, Suzuki T (2017): The change in blood flow and hardness of trapezius muscle after low reactive-level laser irradiation on the stellate ganglion. Journal of Japan Society of Pain Clinicians, 24: 8-11.

21: Masoumipoor M, Jameie SB, Janzadeh A, Nasirinezhad F, Soleimani M, Kerdary M (2014): Effects of 660- and 980-nm low-level laser therapy on neuropathic pain relief following chronic constriction injury in rat sciatic nerve. Lasers Med Sci, 29: 1593-1598. 